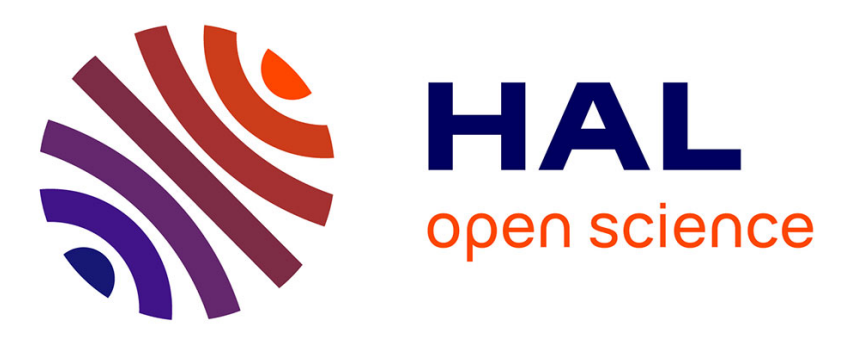

\title{
Calibration procedures for quantitative multiple wavelengths reflectance microscopy
}

Yasmine Fedala, Sorin Munteanu, Frédéric Kanoufi, Gilles Tessier, Jean-Paul Roger, Chang Wu, Fabien Amiot

\section{- To cite this version:}

Yasmine Fedala, Sorin Munteanu, Frédéric Kanoufi, Gilles Tessier, Jean-Paul Roger, et al.. Calibration procedures for quantitative multiple wavelengths reflectance microscopy. Review of Scientific Instruments, 2016, 87 (1), pp.013702. 10.1063/1.4939253 . hal-01480032

\section{HAL Id: hal-01480032 \\ https://hal.science/hal-01480032}

Submitted on 1 Mar 2017

HAL is a multi-disciplinary open access archive for the deposit and dissemination of scientific research documents, whether they are published or not. The documents may come from teaching and research institutions in France or abroad, or from public or private research centers.
L'archive ouverte pluridisciplinaire HAL, est destinée au dépôt et à la diffusion de documents scientifiques de niveau recherche, publiés ou non, émanant des établissements d'enseignement et de recherche français ou étrangers, des laboratoires publics ou privés. 


\section{Calibration procedures for quantitative multiple wavelengths reflectance microscopy}

Yasmina Fedala, ${ }^{1,2}$ Sorin Munteanu, ${ }^{2}$ Frédéric Kanoufi, ${ }^{2}$ Gilles Tessier, ${ }^{1,3}$ Jean Paul

Roger, ${ }^{1}$ Chang $\mathrm{Wu}^{4}{ }^{4}$ and Fabien Amiot ${ }^{4, a)}$

1) Institut Langevin, ESPCI ParisTech / CNRS-UMR 7587, 1 rue Jussieu, 75238 Paris Cedex 05, France

2) PECSA, ESPCI ParisTech / CNRS-UMR 7195, 10 rue Vauquelin, 75231 Paris Cedex 05, France

3) Laboratoire de Neurophotonique, Paris Descartes Univ./ CNRS-UMR 8250, 45 rue des Saints Pères 75270 Paris Cedex 06, France

4) FEMTO-ST Institute, CNRS-UMR 6174 / UBFC / ENSMM / UTBM, 24 chemin de l'Épitaphe, F-25030 Besançon, France

(Dated: 12 January 2016)

In order to characterize surface chemo-mechanical phenomena driving micro-electromechanical systems (MEMS) behavior, it has been previously proposed to use reflected intensity fields obtained from a standard microscope for different illumination wavelengths. Wavelength-dependent and -independent reflectivity fields are obtained from these images provided the relative reflectance sensitivities ratio can be identified. This contribution focuses on the necessary calibration procedures and mathematical methods allowing for a quantitative conversion from a mechanically-induced reflectivity field to a surface rotation field, therefore paving the way for a quantitative mechanical analysis of MEMS under chemical loading.

a)Electronic mail: fabien.amiot@femto-st.fr 


\section{INTRODUCTION}

Because of their high surface over volume ratio, the surface of micrometer sized structures plays a key role in their mechanical behavior, and this property has been proposed to devise micromechanical sensors of environmental changes ${ }^{1}$. A significant effort has been put on the development of biological sensors ${ }^{2}$, but the basic understanding of coupled surface phenomena ${ }^{3}$ has somehow been left behind.

Imaging techniques usually reveal the heterogeneity of surface affinities at the micrometer scale ${ }^{4}$, so that the assessment of the chemical homogeneity of the considered surface is required when focusing on chemo-mechanical coupling phenomena. As surface chemical composition modifications induce (complex) reflection coefficient changes, Jin et al. ${ }^{5}$ proposed an ellipsometric imaging set-up to measure the optical thickness of thin adsorbed films, when Li et al. ${ }^{6}$ use interferometry to measure locally the concentration profiles of reactants near an electrode. Differential reflectance changes detections have been achieved to perform sensitive electroreflectance ${ }^{7}$ and thermoreflectance ${ }^{8}$ measurements, providing access to electrochemically induced effects at substrate-electrolyte interfaces and surface temperature fields.

Focusing on chemo-mechanical coupled phenomena also requires to measure kinematic fields of deformable surfaces. In the field of cantilever sensors, Mertens et $a l^{9}{ }^{9}$ coupled a scanning stage to the standard optical lever technique to measure cantilever sensors profiles. Several interferometric techniques have also been proposed relying on phase changes related to the out-of-plane displacement field ${ }^{10}$. This optical lever technique could also be combined to ellipsometric measurements in order to simultaneously monitor the mean surface curvature and average molecules surface densities on the surface of a microcantilever ${ }^{11}$.

In order to retrieve spatially resolved data, we earlier proposed to use multiple wavelengths imaging reflection microscopy ${ }^{12}$. This technique only requires minor modifications of commercially available reflection microscopes and can provide both local surface modification and kinematic field measurements. A decoupling method has been presented to distinguish wavelength-dependent (e.g., surface changes) and -independent (e.g., kinematic) contributions to the collected intensity. This decoupling method requires the identification of the ratio of the relative reflectance sensitivities for the considered wavelengths. In addition, converting the wavelength-independent contributions into a quantitative surface 
deformation requires a dedicated calibration procedure.

This paper therefore aims at providing the details of robust but simple and straightforward procedures allowing one to retrieve the calibration parameters necessary to decouple wavelength-dependent and wavelength-independent contributions and to provide quantitative kinematic data. The set-up as well as the decoupling procedure are first detailed. The procedure for the identification of the relative reflectance sensitivities ratio is described and simple criteria to assess the identification procedure quality are proposed. The calibration method used to convert the wavelength-independent reflectivity change field into mechanical information is then detailed, and the conversion method is also presented, possibly taking chromatic aberrations into account. Results obtained when investigating the electro-elastic coupling on a microcantilever beam immersed in an electrolyte are used to illustrate the different steps throughout the paper.

\section{MULTIPLE WAVELENGTHS REFLECTANCE MICROSCOPY}

\section{A. Samples}

The deformable mechanical microcantilevers used throughout the paper to exemplify the different calibration and measurement procedures are made of LPCVD silicon nitride whose stoichiometry is adjusted to minimize residual stresses. The considered microcantilever is $431 \mathrm{~nm}$ thick, 80 micrometers long and 15 micrometers wide.

This microcantilever (see Fig.1) is covered with a $10 \mathrm{~nm}$ thick chromium adhesion layer and a gold electrode $(50 \mathrm{~nm})^{13}$. The electrodes are such that each cantilever is electrically addressed independently, and a SU8 insulation layer is used to ensure that only the cantilever surface in addition to a $15 \times 12 \mu \mathrm{m}^{2}$ electrode area on the substrate are in constant contact with the surrounding solution. The temperature of the cantilever is controlled within $0.1^{\circ} \mathrm{C}$ (closed-loop temperature regulation of a Peltier plate) for all the experimental results reported herein.

The described procedures apply to any phenomena modifying the surface reflectivity and this paper presents results obtained when involving the electro-elastic coupling. These are ob-

tained by placing a degassed $\mathrm{KCl} 1 \times 10^{-2} \mathrm{M}$ solution around the cantilever and by controlling the electrode potential (working electrode) with a home-made electrochemical workstation ${ }^{14}$. 


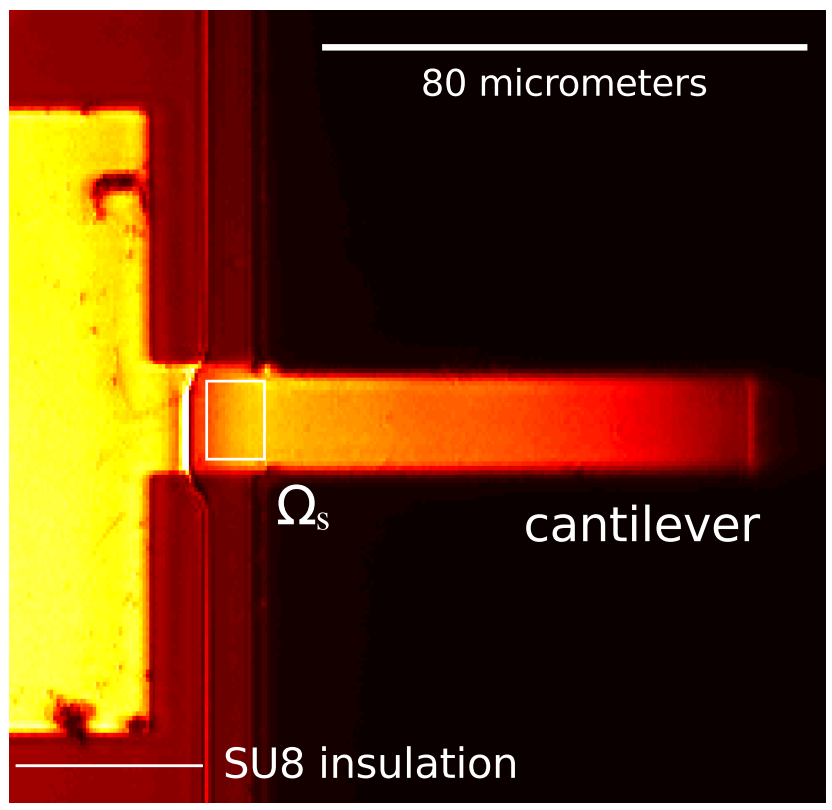

FIG. 1. Optical view of a cantilever beam. $\Omega_{s}$ is the portion of rigid substrate used for calibration (see Sect. III A).

A platinum wire is used as a counter-electrode, and another one is used as a pseudo-reference electrode.

\section{B. Set-up}

The objects are observed with an objective lens (Olympus UMPLFLN $\times 20, N A=0.5$ ) and imaged on a CCD array (Dalsa 1M30, 12 bits, $1024 \times 1024$ pixels) using focusing optics (focal length $200 \mathrm{~mm}$ ) (Fig. 2). In order to distinguish the wavelength-dependent and independent contributions, the sample is illuminated with a Green $\left(\lambda_{G}=530 \mathrm{~nm}\right)$ and a Red $\left(\lambda_{R}=627 \mathrm{~nm}\right)$ light emitting diode (LEDs). These diodes are sequentially triggered and $N$ sums of 10 images are acquired for each illumination wavelength. Building a single sum requires about $1 \mathrm{~s}$.

A particular attention is paid to ensure a non-symmetric, wavelength-independent, light distribution over the pupil of the objective lens. A dichroic mirror is used to similarly illuminate a diaphragm with the two light sources. The image of the diaphragm through a collection lens is placed in the back focal plane of the objective lens (see Fig. 2).

The light distribution is made non-symmetric in the back focal plane of the objective lens 


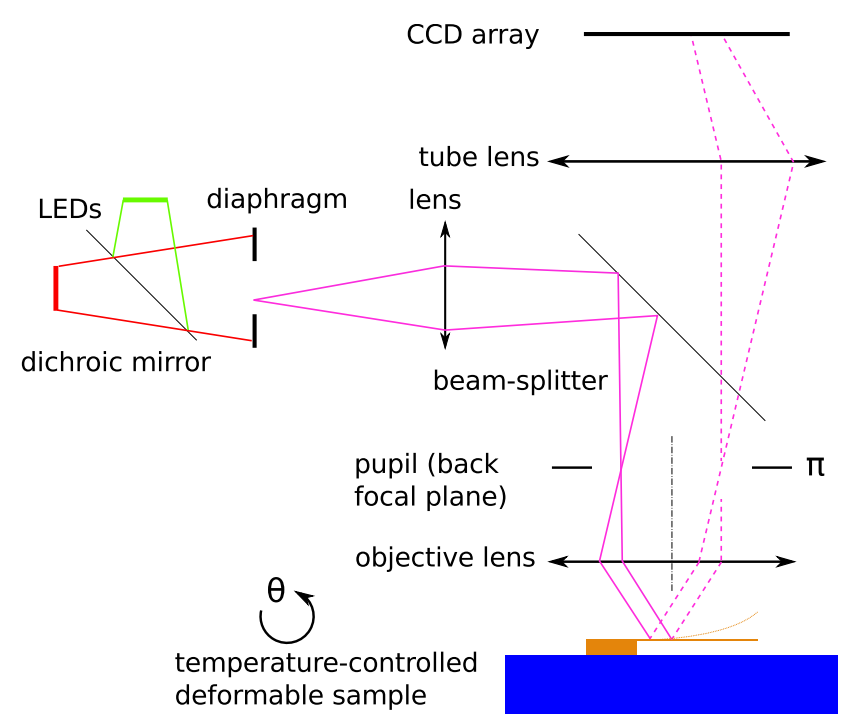

FIG. 2. Schematic of the multiple wavelengths reflection microscope.

by making the image of the diaphragm smaller than the pupil and by shifting this image with respect to the objective lens axis. The image of the diaphragm is thus denoted $D$ in Fig. 3. Assuming that the observed sample is a perfectly flat mirror orthogonal to the objective lens axis, the collected light is distributed over the area $C$ in the back focal plane. Slightly tilting the sample then shifts this area in the back focal plane. If one chooses to move the area $C$ to the area $C_{t}$ (for instance), some of the reflected light is no longer collected by the objective lens (i.e., is cut out by the pupil) and the collected intensity strongly depends on the sample's surface orientation.

Reflected intensity changes may then arise either from surface reflectivity or from collection efficiency changes. The former is usually wavelength-dependent (as with electro- or thermo-reflectance ${ }^{15}$ ) while the latter is (at first glance) wavelength-independent since it primarily depends on the surface orientation. This property is thus exploited in the following to provide both local surface modification and kinematic field measurements. The corresponding formalism previously introduced ${ }^{12}$ is first recalled. The intensity $I_{m}$ collected by the pixel $P$ at wavelength $\lambda$ reads

$$
I_{m}\left(P, \lambda, t_{n}\right)=I_{i}(P, \lambda) R\left(P, \lambda, t_{n}\right) \varepsilon\left(\theta(P), t_{n}\right)
$$

where $I_{i}(P, \lambda)$ is the intensity impinging on the surface element conjugated with pixel $P$, at wavelength $\lambda . R\left(P, \lambda, t_{n}\right)$ is the reflectivity of the surface, i.e., the ratio of the reflected 


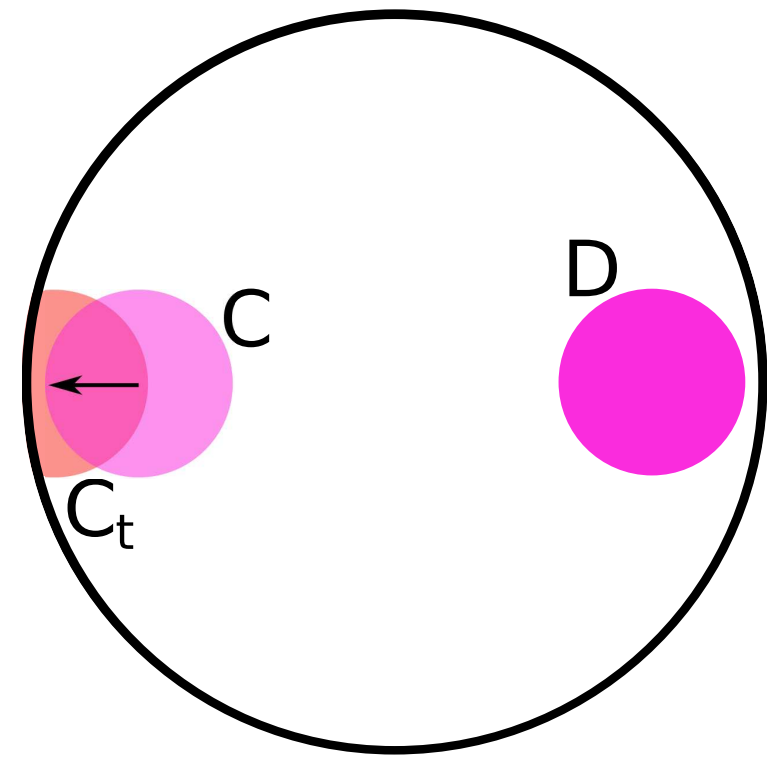

FIG. 3. Representative light distribution in the objective back focal plane $\pi$ (see Fig. 2). $D$ is the image of the illumination diaphragm, $C$ is the collected light distribution for a flat orthogonal mirror. $C_{t}$ is the collected light distribution obtained when tilting such a sample.

and incident intensities, depending on a local driving parameter $X\left(P, t_{n}\right)$ (such as electrical charge density, temperature, etc.) at the time step $t_{n} . \varepsilon\left(\theta(P), t_{n}\right)$ is the collection rate related to the local surface orientation $\theta(P)$. The influence of $X\left(P, t_{n}\right)$ on the reflectivity is assumed to be small, so that $R$ is linearized

$$
R\left(P, \lambda, t_{n}\right)=R_{0}(P, \lambda)\left[1+r(\lambda) X\left(P, t_{n}\right)\right]
$$

with $r(\lambda)=\frac{1}{R_{0}} \frac{\partial R}{\partial X}$ the relative reflectance sensitivity to the driving parameter $X$. The collection rate $\varepsilon\left(\theta(P), t_{n}\right)$ is linearized in the case of small surface rotations around the initial orientation $\theta_{0}(P)$

$$
\begin{aligned}
& \varepsilon\left(\theta(P), t_{n}\right)= \\
& \quad \varepsilon_{0}\left(\theta_{0}(P)\right)\left[1+d_{\theta_{0}}(P)\left(\theta\left(P, t_{n}\right)-\theta_{0}(P)\right)\right]
\end{aligned}
$$

with $d_{\theta_{0}}(P)=\frac{1}{\varepsilon_{0}} \frac{\partial \varepsilon}{\partial \theta}$ the local slope sensitivity. Finally, the measured intensity linearly depends on the wavelength-independent and -dependent relative intensity changes $R_{w i}(P, t)$ and $R_{w d}(\lambda, P, t)$, respectively :

$$
\begin{aligned}
& I_{m}\left(P, \lambda, t_{n}\right)= \\
& \quad I_{a}(P, \lambda)\left[1+R_{w d}\left(\lambda, P, t_{n}\right)+R_{w i}\left(P, t_{n}\right)\right]
\end{aligned}
$$


with

$$
\begin{aligned}
I_{a}(P, \lambda) & =I_{i}(P, \lambda) R_{0}(P, \lambda) \varepsilon_{0}\left(\theta_{0}(P)\right) \\
R_{w d}\left(\lambda, P, t_{n}\right) & =r(\lambda) X\left(P, t_{n}\right) \\
R_{w i}\left(P, t_{n}\right) & =d_{\theta_{0}}(P)\left(\theta\left(P, t_{n}\right)-\theta_{0}(P)\right)
\end{aligned}
$$

For a given $\left(P, t_{n}\right)$, Eq.(4) taken for both analyzed wavelengths are recast as the linear system :

$$
\begin{gathered}
{\left[\begin{array}{c}
I_{m}\left(\lambda_{R}\right)-I_{a}\left(\lambda_{R}\right) \\
I_{m}\left(\lambda_{G}\right)-I_{a}\left(\lambda_{G}\right)
\end{array}\right]=} \\
{\left[\begin{array}{cc}
I_{a}\left(\lambda_{R}\right) & I_{a}\left(\lambda_{R}\right) \\
k I_{a}\left(\lambda_{G}\right) & I_{a}\left(\lambda_{G}\right)
\end{array}\right]\left[\begin{array}{c}
R_{w d}\left(\lambda_{R}\right) \\
R_{w i}
\end{array}\right]}
\end{gathered}
$$

where the ratio $k=r\left(\lambda_{G}\right) / r\left(\lambda_{R}\right)$ is assumed to be different from 1 in order to ensure the system is invertible.

\section{IDENTIFICATION OF THE RELATIVE REFLECTANCE SENSITIVITIES RATIO}

The knowledge of the $k$ value corresponding to the considered physical or chemical phenomena is therefore crucial to a robust decoupling procedure. A method to attain it without any additional experimental effort and without any additional assumption is described in the following. The results illustrating the full procedure are obtained by sweeping the electrode potential $U$ during three cycles between -0.2 and $0.2 \mathrm{~V}$ at $4 \mathrm{mV} . \mathrm{s}^{-1}$ while recording the electrode charge $Q\left(t_{n}\right)=\int X\left(P, t_{n}\right) d S$ ( $d S$ is the elementary surface). $N=44$ sums of 10 images are sequentially acquired for each wavelength during each cycle, and 10 additional sums of 10 images have been acquired before sweeping the potential. Stacks for which (at least) one image is found missing are removed, so that 103 images stacks are kept. The recorded voltammogram is displayed on Fig. 4 and indicates that the electrochemical system stays in the double-layer regime, meaning no chemical reaction (besides adsorption) occurs at the electrode's surface. 


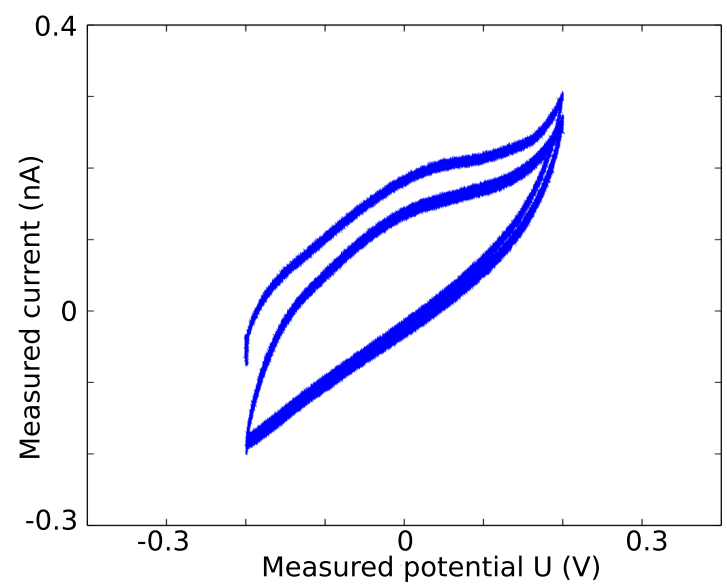

FIG. 4. Recorded current during the three cycles as a function of the imposed potential gap $U$. After the initial cycle, all subsequent voltage cycles lead to identical responses.

\section{A. Identification of the $k$-ratio}

In order to lower the experimental complexity, the proposed procedure makes use of the acquired images, only keeping the red and green intensities collected by pixels corresponding to the part of the electrode on top of the rigid substrate $\left(\Omega_{s}\right)$. This is assumed not to deform during the experiment, so that the recorded reflectivity changes arise only from chemical modifications of the surface. Eq.4 is then recast assuming that

$$
R_{w i}\left(P, t_{n}\right)=0 \forall P \in \Omega_{s}, \forall t_{n}
$$

so that, composing Eq. (4) by ln and assuming small reflectivity changes

$$
\ln \left(I_{m}\left(P, \lambda, t_{n}\right)\right)-\ln \left(I_{a}(P, \lambda)\right) \simeq r(\lambda) X\left(P, t_{n}\right) \forall P \in \Omega_{s}, \forall t_{n}
$$

The equations for the different wavelengths are then easily combined and averaged to cancel the contribution of $I_{a}(P, \lambda)$ :

$$
\mathbf{L}\left(P \in \Omega_{s}, \lambda_{R}\right)-k^{-1} \mathbf{L}\left(P \in \Omega_{s}, \lambda_{G}\right)=\mathbf{0}
$$

where the vector $\mathbf{L}$ is defined, for pixels $P \in \Omega_{s}$, by

$$
\mathbf{L}(P, \lambda)=\ln \left(I_{m}\left(P, \lambda, t_{n}\right)\right)-\left\langle\ln \left(I_{m}\left(P, \lambda, t_{n}\right)\right)\right\rangle
$$

and $\langle\cdot\rangle$ stands for the time average. $\mathbf{L}(P, \lambda)$ therefore features one entry per pixel and per time step. As a consequence of Eq. (11), the matrix $\mathcal{M}_{k}$

$$
\mathcal{M}_{k}=\left[\mathbf{L}\left(P \in \Omega_{s}, \lambda_{R}\right), \mathbf{L}\left(P \in \Omega_{s}, \lambda_{G}\right)\right]
$$


should be singular. A singular value decomposition ${ }^{16}$ (SVD) of $\mathcal{M}_{p}^{t} \mathcal{M}_{p}$ is thus performed to yield

$$
\mathcal{M}_{k}^{t} \mathcal{M}_{k}=\mathcal{V} \mathcal{E} \mathcal{V}^{t}
$$

$\mathcal{V}$ is a $2 \times 2$ orthogonal matrix and $\mathcal{E}$ is a $2 \times 2$ diagonal matrix, with singular values decreasing with the index. The ratio $k$ is therefore obtained as

$$
k=-\frac{\mathcal{V}_{12}}{\mathcal{V}_{22}}
$$

The identification quality can therefore be assessed by considering the ratio $S_{k}=\mathcal{E}_{22} / \mathcal{E}_{11}$ which should vanish. The identification quality may be further assessed by rewriting Eq.(4)

$$
\begin{aligned}
& I_{m}\left(P, \lambda_{R}, t_{n}\right)=I_{a}\left(P, \lambda_{R}\right)\left(1+r\left(P, \lambda_{R}\right) Q\left(t_{n}\right) l(P)\right) \forall P \in \Omega_{s}, \forall t_{n} \\
& I_{m}\left(P, \lambda_{G}, t_{n}\right)=I_{a}\left(P, \lambda_{G}\right)\left(1+k r\left(P, \lambda_{R}\right) Q\left(t_{n}\right) l(P)\right) \forall P \in \Omega_{s}, \forall t_{n}
\end{aligned}
$$

with

$$
X\left(P, t_{n}\right)=Q\left(t_{n}\right) l(P)
$$

$l(P)$ is a localization function relating the local charge density to the overall electrode charge. Setting $k$ to the above-determined value, the system (16) may be solved in a leastsquare sense for $\left[I_{a}\left(P, \lambda_{R}\right), I_{a}\left(P, \lambda_{G}\right), r\left(P, \lambda_{R}\right) l(P) I_{a}\left(P, \lambda_{R}\right), r\left(P, \lambda_{R}\right) l(P) I_{a}\left(P, \lambda_{G}\right)\right]^{t}$ at each location. One defines

$$
\begin{aligned}
\eta_{r}^{2}\left(P, t_{n}\right)= & \frac{\left[I_{m}\left(P, \lambda_{R}, t_{n}\right)-I_{a}\left(P, \lambda_{R}\right)\left(1+r\left(P, \lambda_{R}\right) Q\left(t_{n}\right) l(P)\right)\right]^{2}}{2 \sigma_{R}^{2}} \\
& +\frac{\left[I_{m}\left(P, \lambda_{G}, t_{n}\right)-I_{a}\left(P, \lambda_{G}\right)\left(1+k r\left(P, \lambda_{R}\right) Q\left(t_{n}\right) l(P)\right)\right]^{2}}{2 \sigma_{G}^{2}}
\end{aligned}
$$

where $\sigma_{R}^{2}$ and $\sigma_{G}^{2}$ are the estimated variances on the red and green intensity measurements, respectively. $\eta_{r}^{2}\left(P, t_{n}\right)$ is insensitive to non-uniform charge distribution so that it is possible to further assess the identification quality by plotting the map $\left\langle\eta_{r}^{2}\left(P, t_{n}\right)\right\rangle$, which can then be used to locate areas featuring a modified surface chemical activity (i.e., a different $k$ ). It should be emphasized that this identification procedure does not make use of any assumption regarding the charge distribution over $\Omega_{s}$ (i.e., the localization function $l(P)$ ).

\section{B. Example}

This procedure is applied to the experimental results obtained during the experiment presented above. It should be highlighted that this calibration does not require any additional experimental effort, since it is based on the images acquired to monitor the coupled 
phenomenon under scrutiny. The only requirement is to have optical access to a fixed and rigid part undergoing the same chemical process. In the present case, $\Omega_{s}$ covers $19 \times 17$ pixels. The procedure yields $k=1.18$ and a ratio $S_{k}=0.5$, thereby proving the acceptable identification quality. This is also visually confirmed by plotting $\mathbf{L}\left(P \in \Omega_{s}, \lambda_{R}\right)$ as a function of $\mathbf{L}\left(P \in \Omega_{s}, \lambda_{G}\right)$, which is found to exhibit a linear correlation (see Fig. 5). The

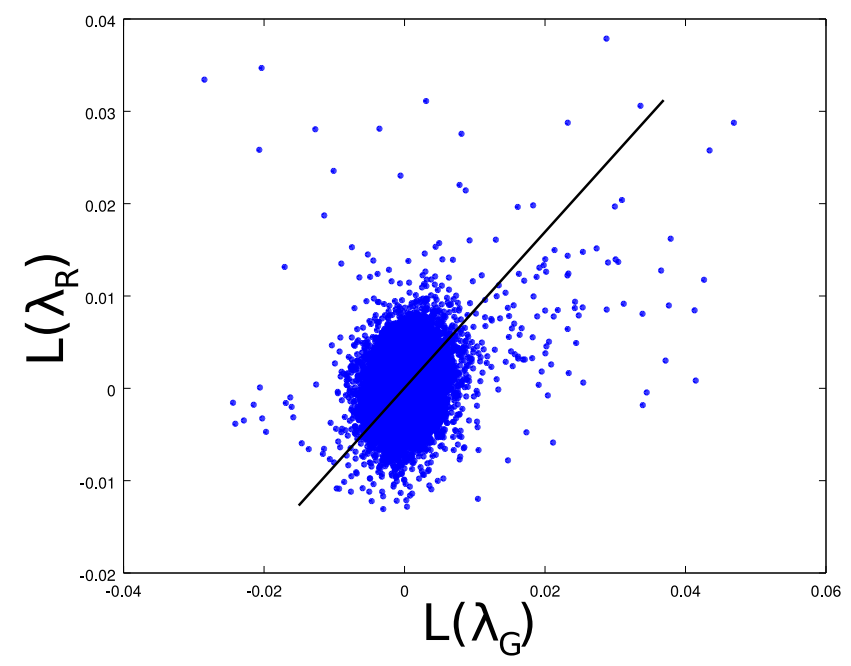

FIG. 5. $\mathbf{L}\left(P \in \Omega_{s}, \lambda_{R}\right)$ as a function of $\mathbf{L}\left(P \in \Omega_{s}, \lambda_{G}\right)$ and the line corresponding to the identified $k$ value.

identification quality may be further assessed by mapping the local residual $\left\langle\eta_{r}^{2}\left(P, t_{n}\right)\right\rangle$ over $\Omega_{s}$ (see Fig. 6). The maximum value corresponds to almost 3 times the variance on the measured intensities and thus remains moderate, thereby proving the considered assumptions are sounded.

\section{IDENTIFICATION OF THE TILT SENSITIVITY}

The use of the decoupling procedure described in section II B together with the $k$ value obtained from the identification procedure detailed in section III A allows one to compute a wavelength-dependent $\left(R_{w d}\left(\lambda_{R}, P, t\right)\right)$ as well as a wavelength-independent $\left(R_{w i}(P, t)\right)$ reflectivity change fields. The former is assumed to describe the spatial distribution of the chemical modifications the surface undergoes, and the latter is assumed to reflect the mechanical deformation of the surface, as detailed in Sect.II B. A calibration procedure exploiting the bimorph effect of the structures under scrutiny is then presented in order to quantitatively 


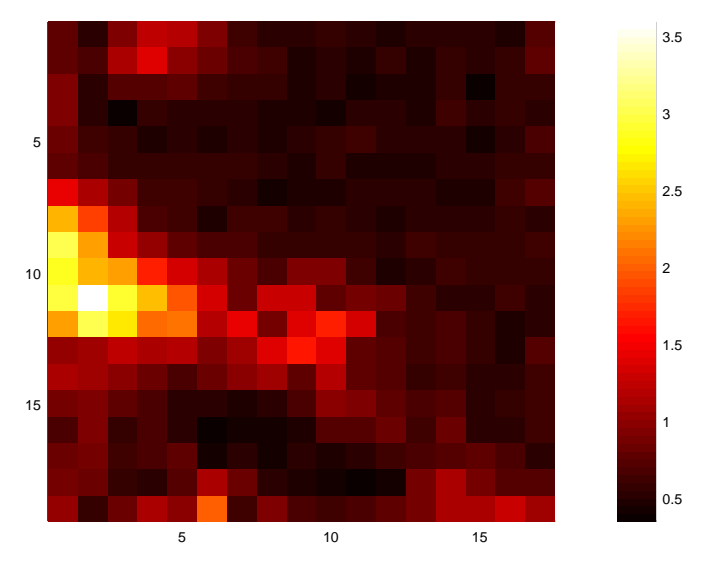

FIG. 6. Normalized residual map $\left\langle\eta_{r}^{2}\left(P, t_{n}\right)\right\rangle$ over the substrate $P \in \Omega_{s}$.

relate the obtained wavelength-independent reflectivity change field to the actual surface rotation field, i.e., to experimentally obtain the tilt sensitivity field $d_{\theta_{0}}(P)$.

\section{A. Thermal calibration procedure}

The proposed calibration procedure makes use of the fact the considered structures are silicon nitride / metal beams and therefore display some bimorph effect. Controlling the temperature is thus a convenient way to prescribe a well known deformation, so that measuring the induced wavelength-independent reflectivity changes allows one to retrieve the map $d_{\theta_{0}}(P)$ defined in Eq. (5). For the present case, the temperature $T$ is varied from $24^{\circ} \mathrm{C}$ to $29^{\circ} \mathrm{C}$ by $0.5^{\circ} \mathrm{C}$ steps, and green and red images are acquired for each step. $N_{T}=15$ images are available for each wavelength.

This temperature changes are assumed to be small enough to neglect thermoreflectance effects $\left(\Delta R / R\right.$ in the $10^{-3}$ range for a $5^{\circ} \mathrm{C}$ temperature change $\left.{ }^{8}\right)$, and the temperature-induced mechanical effect is assumed to be linear with respect to the temperature change :

$$
\begin{array}{r}
I_{m}\left(P, \lambda, T_{n}\right)=I_{a}(P, \lambda)\left[1+d_{\theta_{0}}(P)\left(\theta\left(P, T_{n}\right)-\theta_{0}(P)\right)\right] \\
=I_{a}(P, \lambda)\left[1+d_{\theta_{0}}(P) \beta f(P)\left(T_{n}-T_{0}\right)\right] \\
=I_{a}(P, \lambda)\left[1+\frac{\partial R_{w i}\left(P, T_{0}\right)}{\partial T}\left(T_{n}-T_{0}\right)\right]
\end{array}
$$

$\theta_{0}(P)$ is chosen to represent the mechanical state of the structure in any user-defined reference (temperature) state $T_{0} . \beta$ is a scaling factor and $f(P)$ is the deformation mode. As an 
example, a cantilever beam along the $x$ direction $(x=0$ at the base) subjected to bimetallic effect theoretically yields $f(P)=x \forall x \geqslant 0^{17}$. Denoting $\mathcal{M}_{T}$ the matrix

$$
\mathcal{M}_{T}=\left[\mathbf{1}, \mathbf{T}-T_{0}\right]
$$

The set of Eqs 19 for all temperatures is recast

$$
\mathcal{M}_{T}\left[\begin{array}{c}
I_{a}(P, \lambda) \\
I_{a}(P, \lambda) \frac{\partial R_{w i}\left(P, T_{0}\right)}{\partial T}
\end{array}\right]=\mathbf{I}_{m}(P, \lambda)
$$

The system (21) features $N_{T}$ equations for two unknowns $I_{a}(P, \lambda)$ and $I_{a}(P, \lambda) \frac{\partial R_{w i}\left(P, T_{0}\right)}{\partial T}$ and is thus solved in a least-square sense. The presumably wavelength-independent thermal sensitivity field $\frac{\partial R_{w i}\left(P, T_{0}\right)}{\partial T}$ is then retrieved by forming the ratio of the two unknowns. Fig. 7 thus displays the $\frac{\partial R_{w i}\left(P, T_{0}\right)}{\partial T}$ field, averaged over the cantilever's width, as computed from green and red images, independently.

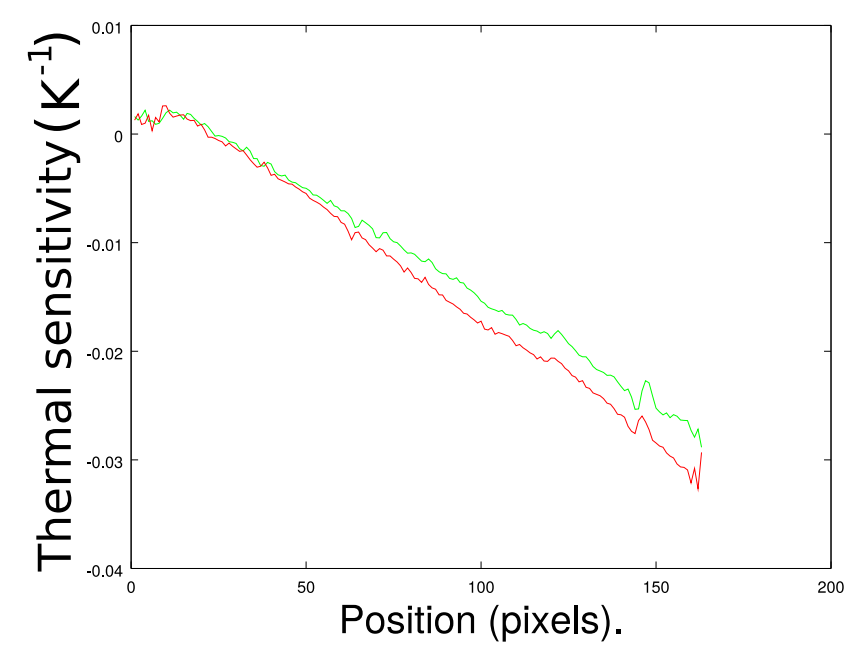

FIG. 7. $\frac{\partial R_{w i}\left(P, T_{0}\right)}{\partial T}$ field, averaged over the cantilever's width, as computed from green (green line) and red images (red line), independently.

The obtained thermal sensitivity fields share the same dependence to the position along the cantilever : the sensitivity is constant (and comparatively small) over $\Omega_{s}$ and varies linearly along the cantilever. Additional experiments (not reported herein) allow to attribute the non-zero sensitivity over $\Omega_{s}$ to a slight out-of-plane chip displacement induced by the temperature change of the sample holder. A slight discrepancy is also observed between the thermal sensitivities computed using green or red images, apparently violating the assumption that a purely mechanical effect induces a wavelength-independent reflectivity change. 


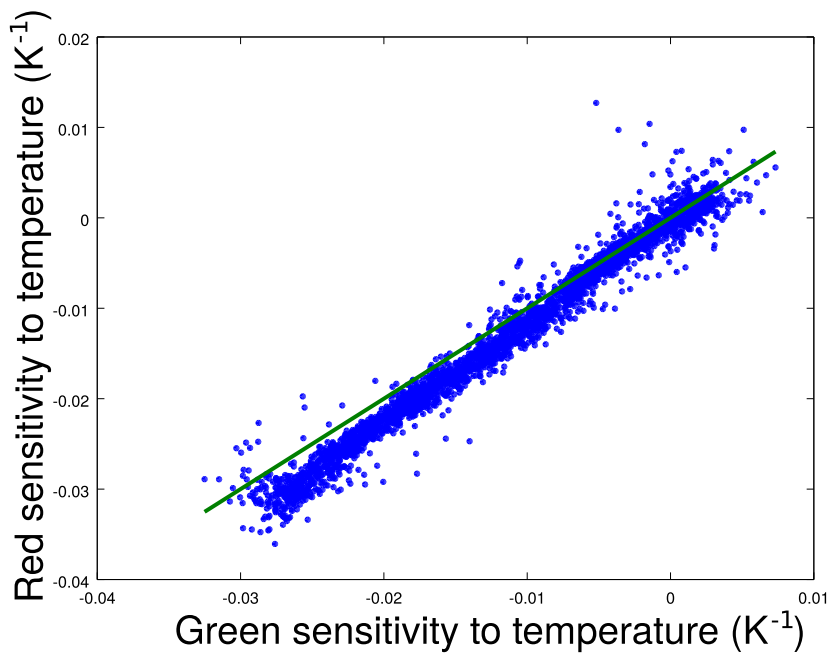

FIG. 8. Red thermal sensitivity as a function of the green thermal sensitivity. The solid line corresponds to $k_{i}=1$.

This is confirmed by plotting the so-computed red thermal sensitivity as a function of the green thermal sensitivity (Fig. 8). These are linearly related but not equal, the equality relation being plotted as a plain line in Fig. 8. Sticking to the formalism defined in Sect. II B, one may define $k_{i}$

$$
k_{i}=\frac{\left(\frac{\partial R_{w i}\left(P, T_{0}\right)}{\partial T}\right)_{G}}{\left(\frac{\partial R_{w i}\left(P, T_{0}\right)}{\partial T}\right)_{R}}
$$

Using the SVD-based estimator described in Sect.III A, one obtains $k_{i}=0.88$ (without correcting for the non-zero sensitivity on the substrate) which has been found to be a very reproducible value for a given objective lens, so that the slight deviation from $k_{i}=1$ is attributed to chromatic aberrations. It should be highlighted that this unexpected behavior does not significantly challenge the approach presented in Sect.II B : Eq. (8) only has to be changed to

$$
\begin{aligned}
& {\left[\begin{array}{c}
I_{m}\left(\lambda_{R}\right)-I_{a}\left(\lambda_{R}\right) \\
I_{m}\left(\lambda_{G}\right)-I_{a}\left(\lambda_{G}\right)
\end{array}\right]=} \\
& {\left[\begin{array}{cc}
I_{a}\left(\lambda_{R}\right) & I_{a}\left(\lambda_{R}\right) \\
k I_{a}\left(\lambda_{G}\right) & k_{i} I_{a}\left(\lambda_{G}\right)
\end{array}\right]\left[\begin{array}{c}
R_{w d}\left(\lambda_{R}\right) \\
R_{w i}\left(\lambda_{R}\right)
\end{array}\right]}
\end{aligned}
$$

and the system is invertible if $k \neq k_{i}$. The mechanically-induced reflectivity change and the local slope sensitivity are thus respectively denoted $R_{w i}\left(\lambda, P, t_{n}\right)$ and $d_{\theta_{0}}(\lambda, P)$ for the sake of generality in the following. 
Inverting Eq.(7) to retrieve the surface rotation from the mechanically-induced reflectivity change requires the knowledge of $d_{\theta_{0}}^{-1}(\lambda, P)$. It should however be highlighted that as one moves from the cantilever tip to the base, one experiences a mechanical effect of decreasing amplitude so that the reflectivity change vanishes close by the cantilever base, as seen from Fig.7 (that is $f(P)$ goes to 0 when moving to the base). As a consequence, determining $d_{\theta_{0}}^{-1}(\lambda, P)$ from $\frac{\partial R_{w i}\left(\lambda, P, T_{0}\right)}{\partial T}$ is expected to fail in a purely local approach (see Eq.19).

The field $d_{\theta_{0}}^{-1}(\lambda, P)$ is thus expanded onto a user-defined basis $\left\{h_{n_{d}}(P)\right\}$ (which is not particularized here for the sake of generality):

$$
d_{\theta_{0}}^{-1}(\lambda, P)=\sum_{n_{d}=0}^{N_{d}} g_{n_{d}}(\lambda) h_{n_{d}}(P)
$$

The coefficients $\left\{g_{n_{d}}(\lambda)\right\}$ may thus be retrieved by solving (see Eq.(19)) :

$$
\beta^{-1} \sum_{n_{d}=0}^{N_{d}} g_{n_{d}}(\lambda) h_{n_{d}}(P) \frac{\partial R_{w i}\left(\lambda, P, T_{0}\right)}{\partial T}=f(P)
$$

Keeping $\left\{g_{n_{d}}\left(\lambda_{R}\right)\right\}$ as unknowns, one has to simultaneously solve

$$
\begin{array}{r}
\beta^{-1} \sum_{n_{d}=0}^{N_{d}} g_{n_{d}}\left(\lambda_{R}\right) h_{n_{d}}(P) \frac{\partial R_{w i}\left(\lambda_{R}, P, T_{0}\right)}{\partial T}=f(P) \\
\beta^{-1} \sum_{n_{d}=0}^{N_{d}} k_{i}^{-1} g_{n_{d}}\left(\lambda_{R}\right) h_{n_{d}}(P) \frac{\partial R_{w i}\left(\lambda_{G}, P, T_{0}\right)}{\partial T}=f(P)
\end{array}
$$

Any considered pixel provides such equalities, so that this equations set is usually overdetermined, since it features only $N_{d}+1$ unknowns. The system (26) is thus solved in a least-square sense to provide the optimal values for the unknowns $\beta^{-1} \tilde{g_{n_{\theta}}}\left(\lambda_{R}\right)$. Assuming the geometry of the cantilever is well known, $\beta$ may be for instance calculated using elasticity theory ${ }^{17}$ to provide the coefficients $\tilde{g}_{n_{\theta}}\left(\lambda_{R}\right)$ and thus the optimal field $d_{\theta_{0}}^{-1}\left(\lambda_{R}, P\right)=$ $\sum_{n_{d}=0}^{N_{d}} \tilde{g_{n_{\theta}}}\left(\lambda_{R}\right) h_{n_{d}}(P)$. $\beta$ may also be experimentally approached, as detailed in next Section.

\section{B. Interferometric reference measurements}

The margins on the dimensions of micrometer-sized devices may however be relatively large, so that this section is intended to provide an experimental procedure to retrieve $\beta$ for the cantilever under scrutiny. The coefficient $\beta$ is obtained prior to the above-described calibration procedure from similar experiments conducted in air with a commercially available 
Mirau interferometric objective lens (Nikon IC Epi Plan DI 10x). An image acquired at a given temperature $T$ results from the phase map $\phi(P, \lambda, T)$ and formally reads

$$
I_{r}(P, \lambda, T)=I_{0 r}(P, \lambda)+A(P, \lambda) \cos [\phi(P, \lambda, T)]
$$

which is recast to highlight the role of the thermally-induced cantilever bending ${ }^{17}$ :

$$
\begin{aligned}
& I_{r}\left(P, \lambda, T_{n}\right)=I_{0 r}(P, \lambda)+ \\
& \quad A(P, \lambda) \cos \left[\phi\left(P, \lambda, T_{0}\right)-\phi\left(P \in \Omega_{s}, \lambda, T_{0}\right)+\frac{2 \pi n \beta\left(T_{n}-T_{0}\right) x^{2}}{\Gamma \lambda}+\phi\left(P \in \Omega_{s}, \lambda, T_{n}\right)\right](28)
\end{aligned}
$$

$n$ is the refractive index of the surrounding medium. $I_{0 r}(P, \lambda)$ and $A(P, \lambda)$ are the mean intensity and contrast fields, respectively. The Mirau objective is illuminated using the same light distribution as in Sect.II B and $\Gamma$ is the correction factor introduced to account for the resulting incidence angle. Denoting

$$
\Delta_{s}\left(\lambda, T_{n}\right)=\phi\left(P \in \Omega_{s}, \lambda, T_{n}\right)-\phi\left(P \in \Omega_{s}, \lambda, T_{0}\right)
$$

Eq.(28) reads

$$
\begin{aligned}
& I_{r}\left(P, \lambda, T_{n}\right)=I_{0 r}(P, \lambda)+ \\
& \quad\left[\cos \left(\Delta_{s}\left(\lambda, T_{n}\right)\right) \cos \left(\frac{2 \pi n \beta\left(T_{n}-T_{0}\right) x^{2}}{\Gamma \lambda}\right)\right. \\
& \left.\quad-\sin \left(\Delta_{s}\left(\lambda, T_{n}\right)\right) \sin \left(\frac{2 \pi n \beta\left(T_{n}-T_{0}\right) x^{2}}{\Gamma \lambda}\right)\right] A(P, \lambda) \cos \phi\left(P, \lambda, T_{0}\right)+ \\
& \quad\left[-\sin \left(\Delta_{s}\left(\lambda, T_{n}\right)\right) \cos \left(\frac{2 \pi n \beta\left(T_{n}-T_{0}\right) x^{2}}{\Gamma \lambda}\right)\right. \\
& \left.\quad-\cos \left(\Delta_{s}\left(\lambda, T_{n}\right)\right) \sin \left(\frac{2 \pi n \beta\left(T_{n}-T_{0}\right) x^{2}}{\Gamma \lambda}\right)\right] A(P, \lambda) \sin \phi\left(P, \lambda, T_{0}\right) \\
& \quad=I_{0 r}(P, \lambda)+ \\
& A(P, \lambda)\left[f_{c}\left(\Delta_{s}\left(\lambda, T_{n}\right), \beta, T_{n}\right) \cos \phi\left(P, \lambda, T_{0}\right)+f_{s}\left(\Delta_{s}\left(\lambda, T_{n}\right), \beta, T_{n}\right) \sin \phi\left(P, \lambda, T_{0}\right)\right\}
\end{aligned}
$$

Again, for a given wavelength, the equations set (30) features $N_{T}$ times the number of pixels equations, for 3 unknowns per pixel (namely, $I_{0 r}, A \cos \phi\left(P, \lambda, T_{0}\right)$ and $A \sin \phi\left(P, \lambda, T_{0}\right)$ ), in addition to the sought parameter $\beta$ and to $N_{T}-1$ phase shifts $\Delta_{s}\left(\lambda, T_{n}\right)$. The system is thus overdetermined, assuming $N_{T}$ is large enough. It is solved in a least-square sense, taking 
advantage of the structure of Eq.(30). The objective function reads

$$
\begin{aligned}
& \eta^{2}\left(I_{0 r}, A \cos \phi\left(P, \lambda, T_{0}\right), A \sin \phi\left(P, \lambda, T_{0}\right), \beta, \Delta_{s}\right)= \\
& \sum_{P, T_{n}}\left\{I_{r}\left(P, \lambda, T_{n}\right)-I_{0 r}(P, \lambda)\right. \\
& -A(P, \lambda)\left[f_{c}\left(\Delta_{s}\left(\lambda, T_{n}\right), \beta, T_{n}\right) \cos \phi\left(P, \lambda, T_{0}\right)\right. \\
& \left.\left.\quad+f_{s}\left(\Delta_{s}\left(\lambda, T_{n}\right), \beta, T_{n}\right) \sin \phi\left(P, \lambda, T_{0}\right)\right]\right\}^{2}
\end{aligned}
$$

The minimization problem is decomposed :

$$
\begin{aligned}
\min _{I_{0 r}, A, \phi\left(P, \lambda, T_{0}\right), \beta, \Delta_{s}} \eta^{2}\left(I_{0 r}, A, \phi\left(P, \lambda, T_{0}\right), \beta, \Delta_{s}\right) & =\min _{\beta, \Delta_{s}} \eta^{2}\left(\tilde{I_{0 r}}, \tilde{A}, \tilde{\phi}\left(P, \lambda, T_{0}\right), \beta, \Delta_{s}\right) \\
& =\min _{\beta} \eta^{2}\left(\tilde{I_{0 r}}, \tilde{A}, \tilde{\phi}\left(P, \lambda, T_{0}\right), \beta, \tilde{\Delta_{s}}\right)
\end{aligned}
$$

$\beta$ and $\Delta_{s}\left(\lambda, T_{n}\right)$ are first initialized, so that the optimal fields $\tilde{I_{0 r}}, \tilde{A} \cos \tilde{\phi}\left(P, \lambda, T_{0}\right)$ and $\tilde{A} \sin \tilde{\phi}\left(P, \lambda, T_{0}\right)$ are easily obtained for any chosen $\beta$ and $\Delta_{s}\left(\lambda, T_{n}\right)$ (linear minimization step). The objective function $\eta^{2}\left(\tilde{I_{0 r}}, \tilde{A}, \tilde{\phi}\left(P, \lambda, T_{0}\right), \beta, \Delta_{s}\right)$ thus only depends on $\beta$ and $\Delta_{s}\left(\lambda, T_{n}\right)$, and has to be minimized with respect to these two variables sets (Eq.(33)). This is achieved by setting the value for $\beta$ and subsequently minimizing the objective function with respect to $\Delta_{s}\left(\lambda, T_{n}\right)$, using a conjugate-gradient algorithm ${ }^{18}$. The resulting objective function thus only depends on $\beta$, and is minimized using the golden-search method ${ }^{18}$, finally yielding an experimental $\tilde{\beta}$ value. Applying this procedure to the data recorded for the

considered cantilever (not shown herein) yields $\tilde{\beta}=-1.49 \times 10^{-5} \mu \mathrm{m}^{-1} K^{-1}$, so that the $d_{\theta_{0}}^{-1}\left(\lambda_{R}, P\right)$ field may be obtained.

Fig.9 displays the obtained inverse tilt sensitivity field $d_{\theta_{0}}^{-1}\left(\lambda_{R}, P\right)$ along the cantilever when using a polynomial basis up to degree 2 for $\left\{h_{n_{d}}(x)\right\}$. This sensitivity, which is found to be rather uniform along the cantilever, is necessary to the conversion into mechanical fields of the measured reflectivity change field (see Eq.7).

\section{CONVERSION FROM THE MECHANICALLY-INDUCED REFLECTIVITY CHANGE FIELD TO KINEMATIC DATA}

This section thus describes a projection procedure used to convert the measured mechanicallyinduced reflectivity change field to a surface rotation field and to filter out noise. 


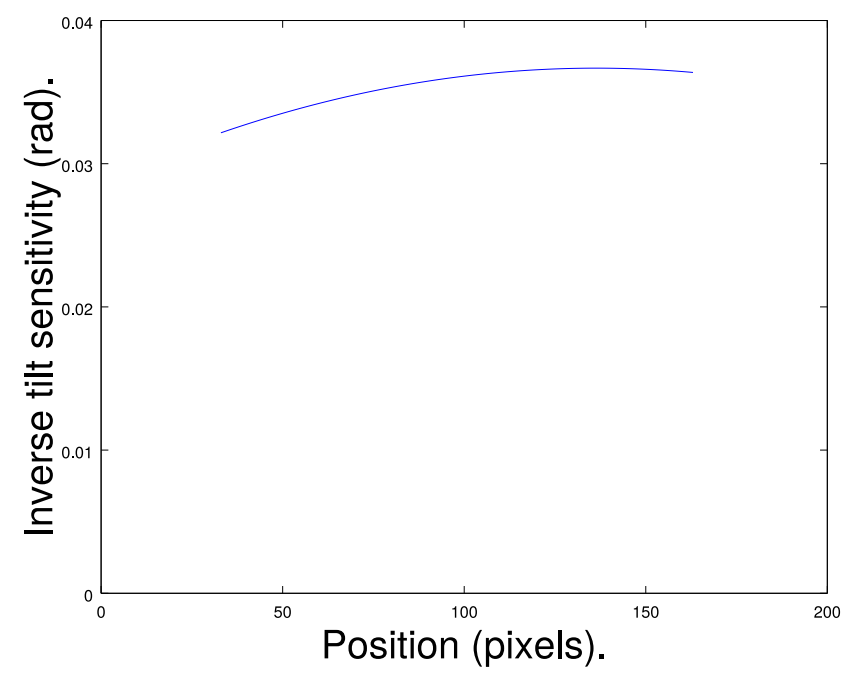

FIG. 9. $d_{\theta_{0}}^{-1}\left(\lambda_{R}, P\right)$ field obtained using a polynomial basis up to degree $2\left(N_{d}=2\right)$.

\section{A. Conversion to kinematic data}

The mechanically-induced contribution may be given a mechanical sense by making use of the $d_{\theta_{0}}^{-1}(P)$ field. The cantilever displacement field $w\left(P, t_{n}\right)$ at time $t_{n}$ may be expanded onto a user-defined functions basis $\left\{\omega_{n_{w}}(P)\right\}$

$$
w\left(P, t_{n}\right)=\sum_{n_{w}=0}^{N_{w}} W_{n_{w}}\left(t_{n}\right) \omega_{n_{w}}(P)
$$

For an Euler-Bernoulli cantilever beam, the surface rotation reads

$$
\begin{aligned}
\theta\left(P, t_{n}\right)-\theta_{0}(P) & =\frac{d w(P)}{d x} \\
& =\sum_{n_{w}=0}^{N_{w}} W_{n_{w}}\left(t_{n}\right) \frac{d \omega_{n_{w}}(P)}{d x}
\end{aligned}
$$

so that the coefficients $W_{n_{w}}\left(t_{n}\right)$ are to be retrieved by projecting the displacement field onto the $\left\{\frac{d \omega_{n_{w}}(P)}{d x}\right\}$ basis using Eq.(7)

$$
\sum_{n_{w}=0}^{N_{w}} W_{n_{w}}\left(t_{n}\right) \frac{d \omega_{n_{w}}(P)}{d x}=d_{\theta_{0}}^{-1}(P) R_{w i}\left(P, t_{n}\right)=\theta\left(P, t_{n}\right)-\theta_{0}(P)
$$

The left-hand side of Eq.(38) is built from the user-defined basis, whereas the right-hand side is the product of the mechanically-induced field obtained in Sect.V B and of the inverse sensitivity obtained in Sect.IV A. There is such an equation for each pixel, for $N_{w}$ unknowns, so that Eq.(38) is an overdetermined linear system. It is thus solved in a least-square sense 
to provide the projection $\Theta\left(P, t_{n}\right)=\sum_{n_{w}=0}^{N_{w}} \tilde{W}_{n_{w}}\left(t_{n}\right) \frac{d \omega_{n_{w}}(P)}{d x}$ of the rotation field onto the chosen basis.

It should be highlighted that this projection of the rotation field onto a functions basis is not strictly necessary (Eq.(7) could be solved for each pixel), but is a convenient and sound way to filter out noise. In addition, $N_{w}$ and $N_{d}$ should not be chosen independently : as seen from Eq.(38), setting $N_{d}>N_{w}$ would filter out terms kept when retrieving $d_{\theta_{0}}^{-1}(P)$ and thus does not make sense.

\section{B. Example}

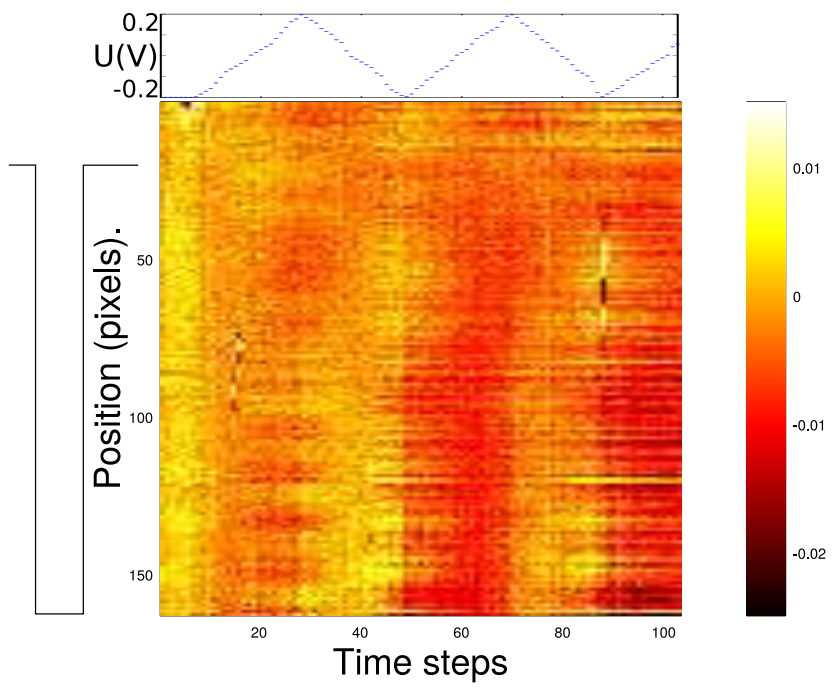

FIG. 10. Identified wavelength-dependent reflectivity change field under cyclic electrochemical actuation, averaged over the cantilever's width $\hat{R}_{w d}\left(\lambda_{R}, x, t_{n}\right)$ (unitless). The cantilever position is schematically drawn on the left, and the actual potential value is recalled on top.

The mechanically-induced $R_{w i}\left(\lambda_{R}, P, t_{n}\right)$ and wavelength-dependent $R_{w d}\left(\lambda_{R}, P, t_{n}\right)$ fields are obtained for the experiment described in Sect.II A and III by solving the linear system (23) for each point and time step, with the parameters identified in Sect.III, IV A. One defines the quantities $\hat{R}_{w d}\left(\lambda_{R}, x, t_{n}\right)$ and $\hat{R}_{w i}\left(\lambda_{R}, x, t_{n}\right)$ as the averages over the cantilever's width of the $R_{w d}\left(\lambda_{R}, P, t_{n}\right)$ and $R_{w i}\left(\lambda_{R}, P, t_{n}\right)$ fields, respectively, in order to allow a 2-D description.

Fig. 10 displays $\hat{R}_{w d}\left(\lambda_{R}, x, t_{n}\right)$, with the cantilever clamping located at $x=16$ pix and the cantilever tip at $x=163$ pix. $\hat{R}_{w d}\left(\lambda_{R}, x, t_{n}\right)$ is mostly about $1 \%$ in magnitude, with a notice- 


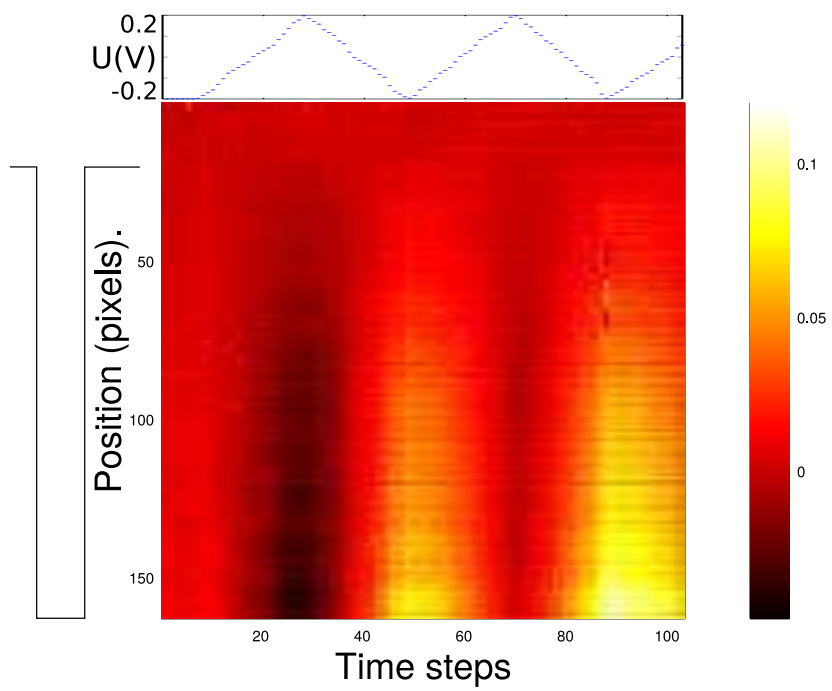

FIG. 11. Identified mechanically-induced reflectivity change field, averaged over the cantilever's width $\hat{R}_{w i}\left(\lambda_{R}, x, t_{n}\right)$ (unitless).

able heterogeneity along the cantilever. It is also seen to be non-zero and electrochemicallydriven on the substrate $(x<16$ pix), thereby proving its non-mechanical origin. This field actually corresponds to the reflectivity field used to monitor surface chemical affinities ${ }^{4}$, and illustrates (wavelength-dependent) electroreflectance phenomena in this particular case ${ }^{19}$. It also shows a slight charge accumulation over the cycles. Fig. 11 shows $\hat{R}_{w i}\left(\lambda_{R}, x, t_{n}\right)$, which is seen to be fairly larger in magnitude (up to 10\%) for the example under scrutiny, owing to the special illumination detailed in Sect.IIB. It is found to have a comparatively very low value on the substrate, which does not exhibit any time dependence, thereby proving its mechanical origin. One should however notice a perturbation around $x=58$ pix at $t_{87}$, which has been found to result from a bubble in the solution traveling above the cantilever.

The raw rotation field $\theta\left(P, t_{n}\right)-\theta_{0}(P)$ and its projection $\Theta\left(P, t_{n}\right)$ onto a polynomial basis (up to degree 3 in $x$ ) are computed according to Eq. (38). Again, one defines the quantities $\hat{\theta}\left(x, t_{n}\right)-\hat{\theta_{0}}(x)$ and $\hat{\Theta}\left(x, t_{n}\right)$ as the averages over the cantilever's width of the $\theta\left(P, t_{n}\right)-\theta_{0}(P)$ and $\Theta\left(P, t_{n}\right)$ fields, respectively.

Fig. 12 shows the raw rotation field averaged over the cantilever's width $\hat{\theta}\left(x, t_{n}\right)-\hat{\theta_{0}}(x)$. It is not computed for locations over the substrate $(x<16$ pix $)$ since the calibration procedure does not deform this surface and thus does not allow for the identification of $d_{\theta_{0}}^{-1}\left(\lambda_{R}, P\right)$ over the substrate (see Fig. 9). The computed $\hat{\theta}\left(x, t_{n}\right)-\hat{\theta}_{0}(x)$ is thus arbitrarily set to 0 for $x<16$ pix in Fig. 12. One should however notice the very good continuity of the rotation at 


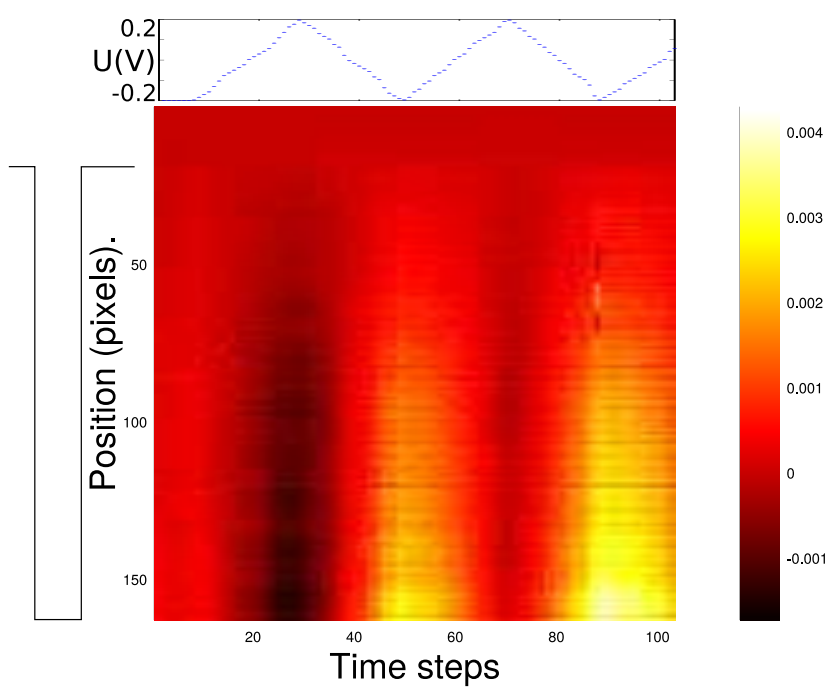

FIG. 12. Raw rotation field, averaged over the cantilever's width $\hat{\theta}\left(x, t_{n}\right)-\hat{\theta_{0}}(x)(\mathrm{rad})$.

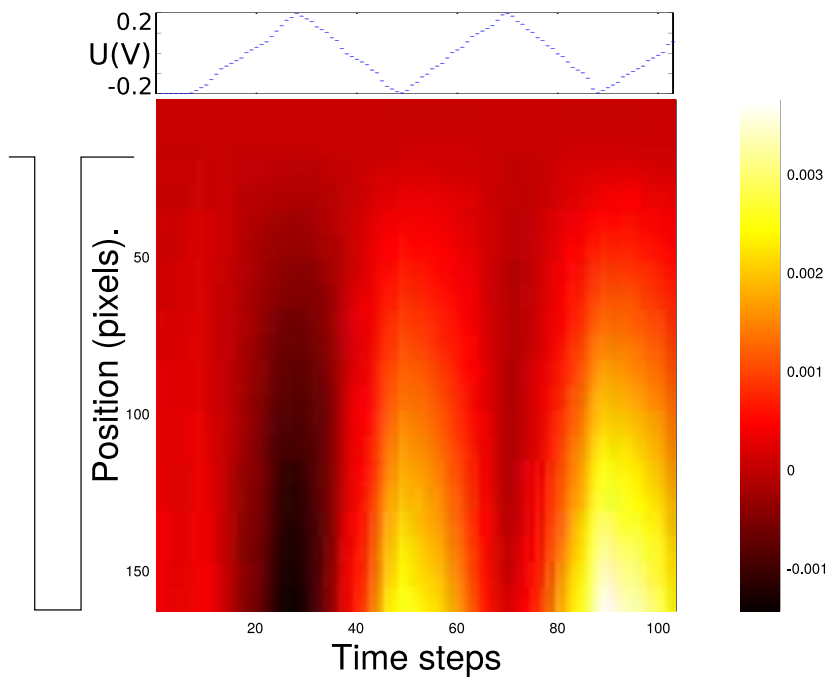

FIG. 13. Projected rotation field, averaged over the cantilever's width $\hat{\Theta}\left(x, t_{n}\right)(\operatorname{rad})$.

$x=16$ pix, reflecting the mechanical nature of $R_{w i}\left(\lambda_{R}, P, t_{n}\right)$. Cycling the potential makes it bend down (away from the electrode side) when the electrode potential is increased, and deformation slightly accumulates across the potential cycles (similarly to the charge density, as seen from Fig.10). Similar results were previously reported with a rather different measurement system ${ }^{20}$. Perturbations on the $R_{w i}\left(\lambda_{R}, P, t_{n}\right)$ field are directly translated to perturbations on the raw rotation field, as seen from $\hat{\theta}\left(x=58\right.$ pix, $\left.t_{87}\right)-\hat{\theta}_{0}(x=58$ pix $)$. This rotation field has been projected for all time steps onto a functional basis containing polynomials up to degree 3 , and its average over the cantilever's width $\hat{\Theta}\left(x, t_{n}\right)$ is displayed in 
Fig. 13. Most of the comments above apply to the projected rotation field. This projection procedure is particularly shown to filter out noise and perturbations, as one could notice from $\hat{\Theta}\left(x=58\right.$ pix, $\left.t_{87}\right)$, which no longer displays any perturbation. One should highlight that having the possibility to choose a functional basis allows one to test for assumptions on the rotation field or to explicitly assume (by using Lagrange multipliers) that the projected rotation field satisfies mechanical constrains (a clamping condition $\Theta\left(x=16\right.$ pix, $\left.t_{n}\right)=0$ for instance).

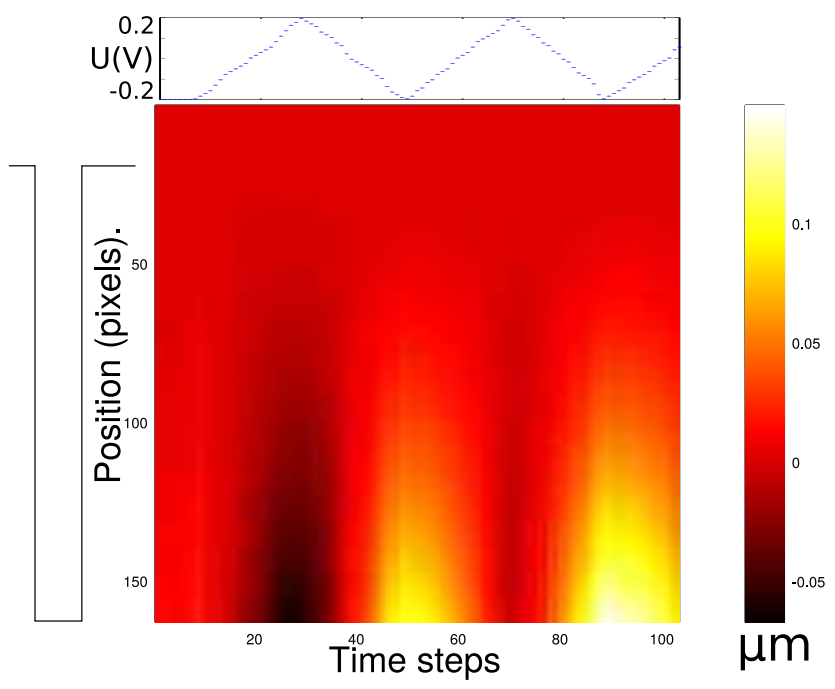

FIG. 14. Displacement field, averaged over the cantilever's width, as obtained by integrating $\hat{\Theta}\left(x, t_{n}\right)($ in $\mu \mathrm{m})$.

The projected rotation field may then be integrated if one wishes to retrieve the out-ofplane displacement field of the cantilever. The result is displayed on Fig. 14 and illustrates the robustness of proposed approach : measuring the surface rotation (instead of the displacement) avoids the use of further derivation (seen as a high-pass filter) in the mechanical exploitation of the data, and one further takes advantage of the projection step to cancel out some measurement noise.

\section{CONCLUSION}

This contribution extensively describes calibration procedures and mathematical methods used to quantitatively translate the fields obtained from multiple-wavelength reflection microscopy with an original illumination device. These procedures are kept minimal by 
making use of the bimorph effect displayed by the gold-coated micromechanical structures extensively used for micromechanical sensors. Most of these calibration procedures may then be automated, keeping the experimental cost minimal. In addition, the decoupling procedure (besides calibration) essentially amounts to solving a linear system. The computation cost is thus also minimal so that (quasi) real-time implementation seem attainable. For systems which do not display any bimetallic effect, any convenient reproducible mechanical actuation may be used, using a similar treatment. The chemo-mechanical phenomena at the origin of the development of cantilever-based sensors may then be thoroughly and quantitatively studied using highly redundant data from a simple commercial reflection microscope featuring minor modifications.

\section{ACKNOWLEDGEMENT}

This work was partially funded through the NRA grants $\mu$ Ecoliers ANR-08-JCJC-0088 (F.K., S.M., Y.F.) and CheMeCo ANR-11-JS09-019-01 (C.W., F.A.). This work was partly supported by the french RENATECH network and its FEMTO-ST technological facility.

\section{REFERENCES}

${ }^{1}$ N. V. Lavrik, M. J. Sepaniak and P. G. Datskos, "Cantilever transducers as a platform for chemical and biological sensors" Rev. Sci. Instrum. 75, 2229 (2004).

${ }^{2}$ J. Fritz, M. K. Baller, H. P. Lang, H. Rothuizen, P. Vettiger, E. Meyer, H.-J. Güntherodt, Ch. Gerber and J. K. Gimzewski, " Translating biomolecular recognition into nanomechanics" Science 288, 316 (2000).

${ }^{3}$ M. F. Hagan, A. Majumdar and A. K. Chakraborty, "Nanomechanical Forces Generated by Surface Grafted DNA" J. Phys. Chem. B 106, 10163 (2002).

${ }^{4}$ S. Munteanu, J.P. Roger, Y. Fedala, F. Amiot, C. Combellas, G. Tessier, F. Kanoufi, "Mapping fluxes of radicals from the combination of electrochemical activation and optical microscopy" Faraday Disc. 164 (1), 241-258 (2013).

${ }^{5}$ G. Jin, P. Tengvall, I. Lundström and H. Arwin, " A biosensor concept based on imaging ellipsometry for visualization of biomolecular interactions" Anal. Biochem. 232, 69 (1995). 
${ }^{6} \mathrm{Q}$. Li and H. S. White, "Interferometric Measurement of Depletion Layer Structure and Voltammetric Data in Concentrated Organic Redox Solutions" Anal. Chem. 67, 561 (1995).

${ }^{7}$ J. Feinleib, "Electroreflectance in metals" Phys. Rev. Lett. 16, 1200 (1966).

${ }^{8}$ G. Tessier, S. Holé and D. Fournier, "Quantitative thermal imaging by synchronous thermoreflectance with optimized illumination wavelengths" Appl. Phys. Lett. 78, 2267 (2001).

${ }^{9}$ J. Mertens, M. Álvarez and J. Tamayo, " Real-time profile of microcantilevers for sensing applications" Appl. Phys. Lett. 87, 234102 (2005).

${ }^{10}$ F. Amiot and JP. Roger, " Nomarski imaging interferometry to measure the displacement field of MEMS" Appl. Opt. 45, 7800 (2006).

${ }^{11}$ M. Godin, O. Laroche, V. Tabard-Cossa, L. Y. Beaulieu, P. Grütter and P. J. Williams, “ Combined in situ Micromechanical Cantilever-Based Sensing and Ellipsometry" Rev. Sci. Instrum. 74, 4902 (2003).

${ }^{12}$ N. Garraud, Y. Fedala, F. Kanoufi, G. Tessier, J.P. Roger and F. Amiot, " Multiple wavelengths reflectance microscopy to study the multi-physical behavior of MEMS" Optics Letters 36 (4), 594-596 (2011).

${ }^{13} \mathrm{C}$. Wu, V. Petrini, E. Joseph and F. Amiot " Design and fabrication of a multiple-thickness electrochemical cantilever sensor" Microelectronic Engineering 19, 1-5 (2014).

${ }^{14}$ A.V. Gopinath and D. Russel "An inexpensive field-portable programmable potentiostat" Chem. Educator 10, 1-6 (2005).

${ }^{15}$ G. Tessier, G. Jerosolimski, S. Holé, D. Fournier and C. Filloy " Measuring and predicting the thermoreflectance sensitivity as a function of wavelength on encapsulated materials" Rev. Sci. Instr. 74, 495 (2003).

${ }^{16}$ J. Demmel and K. Veselic "Jacobi's method is more accurate than QR" SIAM J. Sci. Stat. Comput. 11, 1204-1246 (1992).

${ }^{17}$ S. Timoshenko "Analysis of bi-metal thermostats" J. Opt. Soc. Am. 11(3), 233-255 (1925). ${ }^{18}$ W.H. Press, S.A. Teukolsky, W.T. Vetterling and B.P. Flannery Numerical Recipes in $\mathrm{C}++$ (Cambridge University Press, Cambridge (UK), 2002).

${ }^{19}$ F. Amiot, F. Hild, F. Kanoufi and J. P. Roger "Identification of the electroelastic coupling from full multi-physical fields measured at the micrometre scale" J. Phys. D: Appl. Phys. $403314-3325$ (2007). 
${ }^{20}$ S. Munteanu, S. Gam-Derouich, C. Fammier, Y. Fedala, C. Combellas., F. Amiot and F. Kanoufi "Scanning Electrochemical Microscopy Monitoring in Microcantilever Platforms" Anal. Chem. 84(17), 7449-7455 (2012). 